\title{
Fixed points for modified fuzzy $\psi$-contractive set-valued mappings in fuzzy metric spaces
}

Shihuang Hong*

\section{*Correspondence:}

hongshh@hotmail.com

Institute of Applied Mathematics

and Engineering Computations,

Hangzhou Dianzi University,

Hangzhou, 310018, People's

Republic of China

\section{Springer}

\begin{abstract}
In this paper, we introduce a new concept of fuzzy $\alpha-\psi$-contractive type set-valued mappings and establish fixed-point theorems for such mappings in complete fuzzy metric spaces. Starting from the fuzzy version of the Banach contraction principle, the presented theorems extend, generalize and improve many existing results in the literature. Moreover, the results are supported by examples.
\end{abstract}

Keywords: fixed point; fuzzy contraction; set-valued mapping; fuzzy metric spaces

\section{Introduction}

In metric fixed-point theory, the contractive conditions on underlying functions play an important role for ensuring the existence of fixed points. The Banach contraction principle is a remarkable result in metric fixed-point theory. Recently Gregori and Sapena [1] have extended the Banach contraction principle to fuzzy contractive mappings on complete fuzzy metric spaces in some sense. Over the years, it has been generalized in different directions by several mathematicians (see [2-14]). In particular, Mihet [2] introduced the concepts of fuzzy $\psi$-contractive mappings which enlarge the class of fuzzy contractions in [1], that is, the following implication takes place for the single-valued mapping $T$ :

$$
M(x, y, t)>0 \quad \Rightarrow \quad M(T x, T y, t) \geq \psi(M(x, y, t))
$$

for any $x, y \in X$ and $t>0$, where $\psi$ is a function whose definition is given in Section 3. Moreover, some authors established fixed-point theorems for such mappings in complete fuzzy metric spaces. Afterwards, Hong and Peng [14] modified the notion of the fuzzy $\psi$-contraction via a so-called $f w$-distance $\mathcal{P}$ instead of the fuzzy metric $M$ and provided the sufficient conditions for the existence of fixed points for such contraction set-valued mappings.

Motivated by the works mentioned above, in this paper we will further modify the type of the $\psi$-contraction and establish fixed-point theorems for such set-valued mappings on certain complete fuzzy metric spaces. Specifically, the main purpose is to extend the inequality $M(x, y, t)>0$ to a general functional inequality and introduce therefrom a new called fuzzy $\alpha-\psi$-contraction which extends and improves the fuzzy $\psi$-contraction of setvalued mappings; moreover, to formulate the conditions guaranteeing the convergence of fuzzy $\alpha-\psi$-contractive sequences and the existence of fixed points of such a set-valued mapping. The present fixed-point theorem and a comprehensive set of its corollaries turn

C2014 Hong; licensee Springer. This is an Open Access article distributed under the terms of the Creative Commons Attribution License (http://creativecommons.org/licenses/by/2.0), which permits unrestricted use, distribution, and reproduction in any medium, provided the original work is properly cited. 
out to be generalizations of those of $[1,2,5]$. Some examples are given here to illustrate the usability of the results obtained.

Finally, the idea of present paper has originated from the study of an analogous problem examined by Salimi et al. [15] and Samet et al. [16] for single-valued contractive mappings and Hussain et al. [17] for set-valued contractive mappings on complete determinacy metric spaces.

\section{Preliminaries}

Let us recall [18] that a continuous $t$-norm is a binary operation $*:[0,1] \times[0,1] \rightarrow[0,1]$ such that $([0,1], \leq, *)$ is an ordered Abelian topological monoid with unit 1 . In the sequel, we always assume $a * b \geq a b$ for all $a, b \in(0,1]$.

For examples of a $t$-norm satisfying the above conditions, we enumerate $a * b=a b$, $a * b=\min \{a, b\}$ and $a * b=a b / \max \{a, b, \lambda\}$ for $0<\lambda<1$, respectively.

Definition 2.1 [19] A fuzzy metric space is an ordered triple $(X, M, *)$ such that $X$ is a (nonempty) set, $*$ is a continuous $t$-norm and $M$ is a fuzzy set on $X \times X \times(0,+\infty)$ that satisfies the following conditions, for all $x, y, z \in X$ :

(F1) $M(x, y, t)>0$, for all $t>0$,

(F2) $M(x, x, t)=1$, for all $t>0$, and $M(x, y, t)=1$ for some $t>0$ implies $x=y$,

(F3) $M(x, y, t)=M(y, x, t)$, for all $t>0$,

(F4) $M(x, y, t) * M(y, z, s) \leq M(x, z, t+s)$ for all $s, t>0$ and

(F5) $M(x, y, \cdot):(0,+\infty) \rightarrow[0,1]$ is continuous.

Following the definition of Kramosil and Michálek [20], $M$ is a fuzzy set on $X \times X \times$ $[0, \infty)$ that satisfies (F3) and (F4), while (F1), (F2), (F5) are replaced by (K1), (K2), (K5), respectively, as follows:

(K1) $M(x, y, 0)=0$;

(K2) $M(x, y, t)=1$ for all $t>0$ if and only if $x=y$;

(K5) $M(x, y, \cdot):[0, \infty) \rightarrow[0,1]$ is left continuous.

We refer to these spaces as KM-spaces and refer to the spaces given as in Definition 2.1 as GV-spaces. In addition, when $X$ is called a fuzzy metric space, it means it may be a GV-space or KM-space.

In these senses, $M$ is called a fuzzy metric on $X$. Some simple but useful facts are that

(I) $M(\cdot, \cdot, t)$ is a continuous function on $X \times X$ for $t \in(0, \infty)$ and

(II) $M(x, y, \cdot)$ is nondecreasing for all $x, y \in X$.

Indeed, let $\left\{x_{n}\right\}$ and $\left\{y_{n}\right\}$ be two sequences of $X$ with $\lim _{n \rightarrow \infty} x_{n}=x$ and $\lim _{n \rightarrow \infty} y_{n}=y$. Then, for any $\varepsilon>0$ and $t>0$, we have

$$
M(x, y, t) \geq M\left(x, x_{n}, \varepsilon\right) * M\left(x_{n}, y_{n}, t-2 \varepsilon\right) * M\left(y_{n}, y, \varepsilon\right) .
$$

In view of Lemma 2.2, for any $\delta>0$, we have $M\left(x, x_{n}, \varepsilon\right)>1-\delta$ and $M\left(y_{n}, y, \varepsilon\right)>1-\delta$ for large enough $n$ and any $\varepsilon>0$. Hence,

$$
M(x, y, t) \geq(1-\delta) * M\left(x_{n}, y_{n}, t-2 \varepsilon\right) *(1-\delta) .
$$

Let $\delta \rightarrow 0$; combining the arbitrariness of $\varepsilon$ and the left continuity of $M(x, y, \cdot)$, it follows that

$$
M(x, y, t) \geq 1 * M\left(x_{n}, y_{n}, t\right) * 1=M\left(x_{n}, y_{n}, t\right) .
$$


By taking the limit when $n \rightarrow \infty$, we obtain $\lim _{n \rightarrow \infty} M\left(x_{n}, y_{n}, t\right) \leq M(x, y, t)$. By an analogous inference, we have $M(x, y, t) \geq \lim _{n \rightarrow \infty} M\left(x_{n}, y_{n}, t\right)$. Consequently, $\lim _{n \rightarrow \infty} M\left(x_{n}\right.$, $\left.y_{n}, t\right)=M(x, y, t)$, i.e., the first fact is valid. To prove the second fact, by (F4) we notice that $M(x, y, t) \geq M(x, y, s) * M(y, y, t-s)=M(x, y, s) * 1=M(x, y, s)$ for $s, t \in[0, \infty)$ with $t>s$.

Let $(X, M, *)$ be a fuzzy metric space. For $t>0$ and $r \in(0,1)$, the open ball $B(x, t, r)$ with center $x \in X$ is defined by

$$
B(x, t, r)=\{y \in X: M(x, y, t)>1-r\} .
$$

A subset $A \subset X$ is called open if for each $x \in A$, there exist $t>0$ and $0<r<1$ such that $B(x, t, r) \subset A$. Let $\mathcal{T}$ denote the family of all open subsets of $X$. Then $\mathcal{T}$ is a topology on $X$ induced by the fuzzy metric $M$. This topology is metrizable [21]. Therefore, a closed subset $B$ of $X$ is equivalent to $x \in B$ if and only if there exists a sequence $\left\{x_{n}\right\} \subset B$ such that $\left\{x_{n}\right\}$ topologically converges to $x$. In fact, the topologically convergence of sequences can be indicated by the fuzzy metric as follows.

Lemma 2.2 A sequence $\left\{x_{n}\right\}$ in $X$ is said to be convergent to a point $x \in X$, denoted by $\lim _{n \rightarrow \infty} x_{n}=x$, if $\lim _{n \rightarrow \infty} M\left(x_{n}, x, t\right)=1$ for any $t>0$.

Definition 2.3 [19] Let $(X, M, *)$ be a fuzzy metric space.

(i) A sequence $\left\{x_{n}\right\}$ in $X$ is called Cauchy sequence if for each $\varepsilon>0$ and $t>0$, there exists $n_{0} \in \mathbb{N}$ such that $M\left(x_{n}, x_{m}, t\right)>1-\varepsilon$ for any $m, n \geq n_{0}$.

(ii) A fuzzy metric space $(X, M, *)$ in which every Cauchy sequence is convergent is said to be complete.

There exist two fuzzy versions of Cauchy sequences and completeness, i.e., besides the so-called $M$-Cauchy sequence and $M$-completeness in the sense of Definition 2.3, the G-Cauchy sequence defined by $\lim _{n \rightarrow \infty} M\left(x_{n+p}, x_{n}, t\right)=1$ for all $t, p>0$ and the corresponding $G$-completeness introduced by [22]. In [23] the authors have pointed out that a $G$-Cauchy sequence is not a $M$-Cauchy in general. It is clear that a $M$-Cauchy sequence is $G$-Cauchy and hence a fuzzy metric space is $M$-complete if it is $G$-complete. From now on, by a Cauchy sequence and completeness we mean an $M$-Cauchy sequence and $M$-completeness.

By $C B(X)$ we denote the collection consisting of all nonempty closed subsets of $X$ (obviously, every closed subset of $X$ is bounded in the sense of fuzzy metric spaces). Motivated by [24], we define a function on $C B(X) \times C B(X)$ as follows:

$$
H_{M}(A, B, t)=\min \left\{\inf _{a \in A} M(a, B, t) \inf _{b \in B} M(A, b, t)\right\}
$$

for any $A, B \in C B(X)$ and $t>0$, where $M(c, D, t)=M(D, c, t)=\sup _{d \in D} M(c, d, t)$.

On the collection consisting of compact sunsets of $X$, in [24] the authors have shown that $H_{M}$ satisfies the conditions (F1)-(F5) given as in Definition 2.1. Clearly, $H_{M}(\{x\},\{y\}, t)=$ $M(x, y, t)$ for all $x, y \in X$ and $t>0$.

Lemma 2.4 If $A \subset C B(X)$, then $x \in A$ if and only if $M(x, A, t)=1$ for $t>0$.

We end this section by the following notion which plays an important role in our main results. 
Definition 2.5 Let $(X, M, *)$ be a fuzzy metric space. A subset $D \subset X$ is said to be approximative if the set-valued mapping

$$
\mathcal{P}_{D}(x)=\{y \in D: M(x, y, t)=M(x, D, t), \forall t>0\}, \quad \forall x \in X
$$

has nonempty values. The set-valued mapping $F: X \rightarrow 2^{X}$ is said to have approximative values if $F(x)$ is approximative for each $x \in X$.

It is clear that $F$ has approximative values if it has compact values.

\section{Fixed-point theorems}

Let $(X, M, *)$ be a fuzzy metric space and $T: X \rightarrow C B(X)$ be a set-valued mapping. An element $x \in X$ is called a fixed point of $T$ if $x \in T x$.

Definition 3.1 Let $T: X \rightarrow 2^{X}$ be a set-valued function, and let $\alpha, \eta: X \times X \times(0, \infty) \rightarrow \mathbb{R}_{+}$ be two functions, where $\alpha$ is bounded. We say that $T$ is an $\alpha^{*}-\eta_{*}$-admissible mapping if

$$
\alpha(x, y, t) \leq \eta(x, y, t) \quad \text { implies that } \quad \alpha^{*}(T x, T y, t) \leq \eta_{*}(T x, T y, t), \quad x, y \in X, t>0,
$$

where $\alpha^{*}(A, B, t)=\sup _{x \in A, y \in B} \alpha(x, y, t)$ and $\eta_{*}(A, B, t)=\inf _{x \in A, y \in B} \eta(x, y, t)$.

The following collection $\Psi$ of functions is described in [2], that is, $\psi \in \Psi$ implies that $\psi$ from $[0,1]$ into itself is continuous, nondecreasing, and $\psi(s)>s$ for each $s \in[0,1)$.

Lemma 3.2 Let $\psi \in \Psi$. For every $s>0, \psi(s)>s$ if and only if $\lim _{n \rightarrow \infty} \psi^{n}(s)=1$ uniformly for $s \in[0,1)$, where $\psi^{n}$ is the $n$th iterate of $\psi$.

Proof Necessity. Since $\psi$ is nondecreasing, the sequence $\left\{\psi^{n}(s)\right\}$ is also nondecreasing and hence its limit exists. Let $\lim _{n \rightarrow \infty} \psi^{n}(s)=c$. Thus $c \geq s>0$. If $c<1$, then from the continuity of $\psi$ it follows that

$$
c=\lim _{n \rightarrow \infty} \psi^{n+1}(s)=\psi\left(\lim _{n \rightarrow \infty} \psi^{n}(s)\right)=\psi(c)>c
$$

a contradiction. Therefore, $c=1$.

On the other hand, if the limit is not uniform, then there exists $0<\varepsilon_{0}<1$ such that, for every $n \in \mathbb{N}$, we can find $t_{n} \in(0,1)$ and $k_{n} \geq n$ with $k_{n+1} \geq k_{n}$ satisfying

$$
1>\psi^{k_{n}}\left(t_{n}\right)+\varepsilon_{0} .
$$

We can assume that $t_{n+1} \leq t_{n}$ for $n \in \mathbb{N}$. In fact, if $t_{1}<t_{2}$, then, by means of our assumptions, we have $1>\varepsilon_{0}+\psi^{k_{2}}\left(t_{2}\right) \geq \varepsilon_{0}+\psi^{k_{1}}\left(t_{2}\right) \geq \varepsilon_{0}+\psi^{k_{1}}\left(t_{1}\right)$. Without loss of generality, we put $t_{1}=t_{2}$. Inductively, let $t_{j+1} \leq t_{j}$ for $j>1$. If $t_{j+2}>t_{i}$ for some $i=1,2, \ldots, j+1$, then we have $1>$ $\varepsilon_{0}+\psi^{k_{j+2}}\left(t_{j+2}\right) \geq \varepsilon_{0}+\psi^{k_{j+1}}\left(t_{j+1}\right) \geq \cdots \geq \varepsilon_{0}+\psi^{k_{i}}\left(t_{i}\right)$. In this case, we put $t_{i}=t_{i+1}=\cdots=t_{j+1}=$ $t_{j+2}$. Consequently, the sequence $\left\{t_{n}\right\}$ is nonincreasing and hence is convergent. Denoting $\lim _{n \rightarrow \infty} t_{n}=t_{0}$, we easily see that $1>t_{n} \geq t_{0} \geq 0$. This shows that $\psi^{k_{n}}\left(t_{n}\right) \geq \psi^{k_{n}}\left(t_{0}\right)$. From 
$\lim _{n \rightarrow \infty} \psi^{k_{n}}\left(t_{0}\right)=1$ it follows that $\lim _{n \rightarrow \infty} \psi^{k_{n}}\left(t_{n}\right)=1$. Hence, there exists $k_{0} \in \mathbb{N}$ such that $\psi^{k_{n}}\left(t_{n}\right)>1-\varepsilon_{0} / 2$, for all $n \geq k_{0}$, that is,

$$
1-\varepsilon_{0}>\psi^{k_{n}}\left(t_{n}\right)>1-\varepsilon_{0} / 2
$$

a contradiction. Therefore, $\lim _{n \rightarrow \infty} \psi^{n}(s)=1$ uniformly for $s \in[0,1)$.

Sufficiency. Assume that there exists $t_{0} \in(0,1)$ such that $\psi\left(t_{0}\right) \leq t_{0}$. Then $\psi^{n}\left(t_{0}\right) \leq t_{0}$ for all $n \in \mathbb{N}$ since $\psi$ is nondecreasing. Thus $1=\lim _{n \rightarrow \infty} \psi^{n}\left(t_{0}\right) \leq t_{0}<1$, a contradiction.

Definition 3.3 Let $\psi \in \Psi$. The set-valued mapping $T$ is called a fuzzy $\alpha-\psi$-contractive mapping if the following implication takes place:

$$
x, y \in X, t>0, \quad \alpha(x, y, t) \leq \eta(x, y, t) \quad \Rightarrow \quad H_{M}(T x, T y, t) \geq \psi(N(x, y, t)),
$$

where $\psi \in \Psi$ and

$$
N(x, y, t)=\min \{M(x, y, t), \sqrt{M(x, T x, t) M(y, T y, t)}\} .
$$

Theorem 3.4 Let $(X, M, *)$ be a complete fuzzy metric space and $T: X \rightarrow C B(X)$ be a fuzzy $\alpha-\psi$-contractive and $\alpha^{*}-\eta_{*}$-admissible set-valued mapping. Suppose that the following assertions hold:

(i) there exist $x_{0} \in X$ and $x_{1} \in \mathcal{P}_{T x_{0}}\left(x_{0}\right)$ such that $\alpha\left(x_{0}, x_{1}, t\right) \leq \eta\left(x_{0}, x_{1}, t\right)$ for each $t>0$;

(ii) for any sequence $\left\{x_{n}\right\} \subset X$ converging to $x \in X$ and $\alpha\left(x_{n}, x_{n+1}, t\right) \leq \eta\left(x_{n}, x_{n+1}, t\right)$, for all $n \in \mathbb{N}$ and $t>0$, we have $\alpha\left(x_{n}, x, t\right) \leq \eta\left(x_{n}, x, t\right)$ for all $n \in \mathbb{N}$.

Then $T$ has a fixed point.

Proof Our assumptions guarantee that there exist $x_{0} \in X$ and $x_{1} \in \mathcal{P}_{T x_{0}}\left(x_{0}\right)$ such that $\alpha\left(x_{0}, x_{1}, t\right) \leq \eta\left(x_{0}, x_{1}, t\right)$ and $M\left(x_{0}, x_{1}, t\right)=M\left(x_{0}, T x_{0}, t\right)$ for each $t>0$. By the contractive condition (1) we have

$$
H_{M}\left(T x_{0}, T x_{1}, t\right) \geq \psi\left(N\left(x_{0}, x_{1}, t\right)\right)
$$

for all $t>0$. Noting that $T$ is an $\alpha^{*}-\eta_{*}$-admissible mapping, we have

$$
\alpha^{*}\left(T x_{0}, T x_{1}, t\right) \leq \eta_{*}\left(T x_{0}, T x_{1}, t\right) .
$$

For $x_{1} \in T x_{0}$, there exists $x_{2} \in \mathcal{P}_{T x_{1}}\left(x_{1}\right)$ such that $\alpha\left(x_{1}, x_{2}, t\right) \leq \eta\left(x_{1}, x_{2}, t\right)$. Applying again the contractive condition (1) we have

$$
H_{M}\left(T x_{1}, T x_{2}, t\right) \geq \psi\left(N\left(x_{1}, x_{2}, t\right)\right) .
$$

Continuing this process, we can define a sequence $\left\{x_{n}\right\}$ in $X$ by $x_{n} \in \mathcal{P}_{T x_{n-1}}\left(x_{n-1}\right)$ satisfying, for all $n \in \mathbb{N}$ and $t>0$,

$$
\begin{aligned}
& H_{M}\left(T x_{n}, T x_{n+1}, t\right) \geq \psi\left(N\left(x_{n}, x_{n+1}, t\right)\right), \\
& \alpha\left(x_{n}, x_{n+1}, t\right) \leq \eta\left(x_{n}, x_{n+1}, t\right), \\
& M\left(x_{n}, x_{n+1}, t\right)=M\left(x_{n}, T x_{n}, t\right) .
\end{aligned}
$$


If $x_{n+1}=x_{n}$ for some $n \in \mathbb{N}$, then $x=x_{n}$ is a fixed point of $T$ and the result is proved. Hence, we suppose that $x_{n+1} \neq x_{n}$, i.e., $x_{n} \notin T x_{n}$ for all $n \in \mathbb{N}$. From equation (5) and the definition of $H_{M}$ it follows that

$$
M\left(x_{n}, x_{n+1}, t\right) \geq H_{M}\left(T x_{n-1}, T x_{n}, t\right)
$$

for all $n \in \mathbb{N}$ and $t>0$. By means of equation (3) we have

$$
M\left(x_{n}, x_{n+1}, t\right) \geq \psi\left(N\left(x_{n-1}, x_{n}, t\right)\right) \quad \text { for all } n \in \mathbb{N} \text { and } t>0
$$

On the other hand, by equation (5) we get

$$
\begin{aligned}
N\left(x_{n-1}, x_{n}, t\right) & =\min \left\{M\left(x_{n-1}, x_{n}, t\right), \sqrt{M\left(x_{n-1}, T x_{n-1}, t\right) M\left(x_{n}, T x_{n}, t\right)}\right\} \\
& =\min \left\{M\left(x_{n-1}, x_{n}, t\right), \sqrt{M\left(x_{n-1}, x_{n}, t\right) M\left(x_{n}, x_{n+1}, t\right)}\right\} .
\end{aligned}
$$

By equation (6), this implies that

$$
M\left(x_{n}, x_{n+1}, t\right) \geq \psi\left(\min \left\{M\left(x_{n-1}, x_{n}, t\right), \sqrt{M\left(x_{n-1}, x_{n}, t\right) M\left(x_{n}, x_{n+1}, t\right)}\right\}\right)
$$

for all $n \in \mathbb{N}$ and $t>0$. We claim that

$$
M\left(x_{n-1}, x_{n}, t\right) \leq M\left(x_{n}, x_{n+1}, t\right) \quad \text { for all } n \in \mathbb{N} \text { and } t>0 .
$$

Suppose the contrary; then

$$
\min \left\{M\left(x_{n-1}, x_{n}, t\right), \sqrt{M\left(x_{n-1}, x_{n}, t\right) M\left(x_{n}, x_{n+1}, t\right)}\right\}=\sqrt{M\left(x_{n-1}, x_{n}, t\right) M\left(x_{n}, x_{n+1}, t\right)} .
$$

By virtue of the properties of $\psi$, for all $n \in \mathbb{N}$ and $t>0$, we get

$$
\begin{aligned}
M\left(x_{n}, x_{n+1}, t\right) & \geq \psi\left(\sqrt{M\left(x_{n-1}, x_{n}, t\right) M\left(x_{n}, x_{n+1}, t\right)}\right) \\
& >\sqrt{M\left(x_{n-1}, x_{n}, t\right) M\left(x_{n}, x_{n+1}, t\right)}>M\left(x_{n}, x_{n+1}, t\right),
\end{aligned}
$$

a contradiction. Hence equation (7) is valid. Moreover, in view of the monotonicity of $\psi$ one has

$$
M\left(x_{n}, x_{n+1}, t\right) \geq \psi\left(M\left(x_{n-1}, x_{n}, t\right)\right)>M\left(x_{n-1}, x_{n}, t\right)
$$

for all $n \in \mathbb{N}$ and $t>0$. Repeating this procedure, we have

$$
M\left(x_{n}, x_{n+1}, t\right) \geq \psi\left(M\left(x_{n-1}, x_{n}, t\right)\right) \geq \cdots \geq \psi^{n}\left(M\left(x_{0}, x_{1}, t\right)\right)
$$

for all $n \in \mathbb{N}$ and $t>0$. Now, for all $m>n, m, n \in \mathbb{N}$ and $t>0$, we can write

$$
\begin{aligned}
M\left(x_{n}, x_{m}, t\right) & \geq M\left(x_{n}, x_{n+1}, t_{n+1}\right) * M\left(x_{n+1}, x_{n+2}, t_{n+2}\right) * \cdots * M\left(x_{m-1}, x_{m}, t_{m}\right) \\
& \geq \prod_{i=n}^{m-1} M\left(x_{i}, x_{i+1}, t_{i+1}\right) \geq \prod_{i=n}^{m-1} \psi^{i}\left(M\left(x_{0}, x_{1}, t_{i+1}\right)\right)
\end{aligned}
$$


where $t_{i}>0(i=n+1, n+2, \ldots, m)$ and $\sum_{i=n+1}^{m} t_{i}=t$. In the light of Lemma 3.2, we can assume that $\psi^{i}\left(M\left(x_{0}, x_{1}, t_{i}\right)\right)>1-1 / 2^{i}$ when $i$ is large enough. Note that the series $\sum_{i=1}^{\infty} 1 / 2^{i}$ is convergent, and the infinite product $\prod_{i=1}^{\infty}\left(1-\left(1 / 2^{i}\right)\right)$ is convergent, too. Hence, $\lim _{n \rightarrow \infty} \prod_{i=n}^{\infty}\left(1-\left(1 / 2^{i}\right)\right)=1$. This implies that $\left\{x_{n}\right\}$ is an $M$-Cauchy sequence.

In view of the completeness of $(X, M, *)$, there exists $y \in X$ such that $x_{n} \rightarrow y$ as $n \rightarrow \infty$. By means of (ii), we have $\alpha\left(x_{n}, y, t\right) \leq \eta\left(x_{n}, y, t\right)$ for all $n \in \mathbb{N}$ and $t>0$. From the contractive condition it follows that

$$
H_{M}\left(T x_{n}, T y, t\right) \geq \psi\left(N\left(x_{n}, y, t\right)\right) .
$$

We observe that $x_{n} \notin T x_{n}$, so $M\left(x_{n}, T x_{n}, t\right)<1$ by Lemma 2.4 . If $M(y, T y, t)<1$, then

$$
N\left(x_{n}, y, t\right)=\min \left\{M\left(x_{n}, y, t\right), \sqrt{M\left(x_{n}, T x_{n}, t\right) M(y, T y, t)}\right\}<1 .
$$

Therefore, $H_{M}\left(T x_{n}, T y, t\right) \geq \psi\left(N\left(x_{n}, y, t\right)\right)>N\left(x_{n}, y, t\right)$ for $n \in \mathbb{N}$ and $t>0$. Moreover, by equation (5) we obtain

$$
M\left(x_{n+1}, T y, t\right) \geq H_{M}\left(T x_{n}, T y, t\right)>N\left(x_{n}, y, t\right) .
$$

Noting that $M\left(x_{n}, y, t\right) \rightarrow 1, M\left(x_{n}, T x_{n}, t\right)=M\left(x_{n}, x_{n+1}, t\right) \rightarrow 1$, we have $N\left(x_{n}, y, t\right) \rightarrow$ $\sqrt{M(y, T y, t)}$ as $n \rightarrow \infty$. By taking the limit as $n \rightarrow \infty$ in the above inequality, we obtain

$$
M(y, T y, t) \geq \sqrt{M(y, T y, t)} .
$$

This is a contradiction. Therefore, $M(y, T y, t)=1$. From Lemma 2.4 it follows that $y \in T y$, i.e. $y$ is a fixed point of $T$.

We present the following interesting corollaries.

Corollary 3.5 Let $(X, M, *)$ be a complete fuzzy metric space and let $T$ be an $\alpha^{*}-\eta_{*}$ admissible set-valued mapping with $\eta=1$. Assume that the following assertions hold:

(i) for $\psi \in \Psi, x, y \in X$ and $t>0$,

$$
\alpha(x, y, t) \leq 1 \quad \Rightarrow \quad H_{M}(T x, T y, t) \geq \psi(N(x, y, t))
$$

(ii) there exist $x_{0} \in X$ and $x_{1} \in \mathcal{P}_{T x_{0}}\left(x_{0}\right)$ such that $\alpha\left(x_{0}, x_{1}, t\right) \leq 1$ for each $t>0$;

(iii) for any sequence $\left\{x_{n}\right\} \subset X$ converging to $x \in X$ and $\alpha\left(x_{n}, x_{n+1}, t\right) \leq 1$, for all $n \in \mathbb{N}$ and $t>0$, we have $\alpha\left(x_{n}, x, t\right) \leq 1$ for all $n \in \mathbb{N}$ and $t>0$.

Then $T$ has a fixed point.

Remark 3.6 Let $\alpha(x, y, t)=M(x, y, t)$. Then the assumptions (i) and (ii) of Corollary 3.5 hold and $\alpha(x, y, t) \leq 1$ for all $x, y \in X$ and $t>0$. Thus $H_{M}(T x, T y, t) \geq \psi(N(x, y, t))$ for all $x, y \in X$ and $t>0$ with $\psi \in \Psi$ (here $T$ is called a fuzzy $\psi$-contractive set-valued mapping). Therefore, $T$ has a fixed point in $X$. This result includes Theorem 3.1 in [2] and Theorem 3 in [5], and also the corresponding results of $[1,4]$ in complete GV-spaces. 
Corollary 3.7 Let all hypotheses of Corollary 3.5 hold except (i) changed into one of the following conditions:

(I) for $\psi \in \Psi, x, y \in X$ and $t>0$,

$$
\alpha(x, y, t) H_{M}(T x, T y, t) \geq \psi(N(x, y, t))
$$

(II) for $\psi \in \Psi, x, y \in X$ and $t>0$,

$$
(\alpha(x, y, t)+\lambda)^{H_{M}(T x, T y, t)} \geq(1+\lambda)^{\psi(N(x, y, t))}, \quad \lambda>0 ;
$$

(III) for $\psi \in \Psi, x, y \in X$ and $t>0$,

$$
\left(H_{M}(T x, T y, t)+\lambda\right)^{\alpha(x, y, t)} \geq \psi(N(x, y, t))+\lambda, \quad \lambda>0 .
$$

Then T has a fixed point.

Corollary 3.8 Let $(X, M, *)$ be a complete fuzzy metric space and let $T$ be an $\alpha^{*}-\eta_{*}$ admissible set-valued mapping with $\alpha=1$. Assume that the following assertions hold:

(i) for $\psi \in \Psi, x, y \in X$ and $t>0$,

$$
\eta(x, y, t) \geq 1 \quad \Rightarrow \quad H_{M}(T x, T y, t) \geq \psi(N(x, y, t))
$$

(ii) there exist $x_{0} \in X$ and $x_{1} \in \mathcal{P}_{T x_{0}}\left(x_{0}\right)$ such that $\eta\left(x_{0}, x_{1}, t\right) \geq 1$ for each $t>0$;

(iii) for any sequence $\left\{x_{n}\right\} \subset X$ converging to $x \in X$ and $\eta\left(x_{n}, x_{n+1}, t\right) \geq 1$, for all $n \in \mathbb{N}$ and $t>0$, we have $\eta\left(x_{n}, x, t\right) \geq 1$ for all $n \in \mathbb{N}$.

Then $T$ has a fixed point.

\section{Examples}

In this section, we conclude the paper with several examples to illustrate the usability of the obtained results.

Example 4.1 Let $X=[0, \infty), a * b=a b$ for any $a, b \in[0,1]$ and $M(x, y, t)=\frac{\min \{x, y\}}{\max \{x, y\}}$ for $x, y \in X$ and $t>0$. Then, for given $\lambda>0$, the set-valued mapping $T: X \rightarrow X$, where

$$
T x= \begin{cases}\{x+\lambda\}, & x+\lambda<1, \\ {[\sqrt{x}, \sqrt{x+\lambda}],} & x+\lambda \geq 1,\end{cases}
$$

has a fixed point in $X$.

Proof Using similar arguments to the ones in [25, Theorem 16], one shows that $(X, M, *)$ is a complete GV-space. Let $\psi(t)=\sqrt{t}$ for $t \in[0,1]$. Then $\psi \in \Psi$. Let

$$
\alpha(x, y, t)=\left\{\begin{array}{ll}
2, & x \text { or } y \in[0,1), \\
\frac{1}{2}, & \text { otherwise, }
\end{array} \quad x, y \in X, t>0 .\right.
$$

Then $\alpha(x, y, t) \leq 1$ implies that $x, y \in[1, \infty)$. For any $x, y \in X$ and $t>0$, we consider the following cases. 
Case 1. $\sqrt{x+\lambda}<\sqrt{y}$. In this case, we have, for any $a \in T x, M(a, T y, t)=\frac{a}{\sqrt{y}}$ and $\inf _{a \in T x} M(a, T y, t)=\frac{\sqrt{x}}{\sqrt{y}}$. Similarly, $\inf _{b \in T y} M(T x, b, t)=\frac{\sqrt{x+\lambda}}{\sqrt{y+\lambda}}$. Consequently, we obtain

$$
H_{M}(T x, T y, t)=\min \left\{\frac{\sqrt{x}}{\sqrt{y}}, \frac{\sqrt{x+\lambda}}{\sqrt{y+\lambda}}\right\}=\frac{\sqrt{x}}{\sqrt{y}}=\psi(M(x, y, t)) .
$$

Case 2. $\sqrt{x}<\sqrt{y} \leq \sqrt{x+\lambda}$. We get $\inf _{a \in T x} M(a, T y, t)=\frac{\sqrt{x}}{\sqrt{y}}$ and $\inf _{b \in T y} M(T x, b, t)=\frac{\sqrt{x+\lambda}}{\sqrt{y+\lambda}}$. Consequently,

$$
H_{M}(T x, T y, t)=\min \left\{\frac{\sqrt{x}}{\sqrt{y}}, \frac{\sqrt{x+\lambda}}{\sqrt{y+\lambda}}\right\}=\frac{\sqrt{x}}{\sqrt{y}}=\psi(M(x, y, t)) .
$$

Case 3. $\sqrt{y} \leq \sqrt{x}<\sqrt{y+\lambda}$ or $\sqrt{y+\lambda} \leq \sqrt{x}$. We get $\inf _{b \in T y} M(T x, b, t)=\frac{\sqrt{y}}{\sqrt{x}}$ and $\inf _{a \in T x} M(a, T y, t)=\frac{\sqrt{y+\lambda}}{\sqrt{x+\lambda}}$. Consequently,

$$
H_{M}(T x, T y, t)=\min \left\{\frac{\sqrt{y}}{\sqrt{x}}, \frac{\sqrt{y+\lambda}}{\sqrt{x+\lambda}}\right\}=\frac{\sqrt{y}}{\sqrt{x}}=\psi(M(x, y, t)) .
$$

This shows that $\alpha$ and $T$ satisfy Corollary 3.5(i). Notice that $\sqrt{2+\lambda} \in T \sqrt{2}$ and $\alpha(\sqrt{2}$, $\sqrt{2+\lambda}, t)=\frac{1}{2}<1$; that is, Corollary 3.5(ii) is satisfied.

Now, if $\left\{x_{n}\right\}$ is a sequence in $X$ such that $\alpha\left(x_{n}, x_{n+1}, t\right) \leq 1$, for all $t>0$ and $n \in \mathbb{N}$, and $x_{n} \rightarrow x$ as $n \rightarrow \infty$, then $\left\{x_{n}\right\} \subset[1, \infty)$, which implies that $x \geq 1$. This guarantees that $\alpha\left(x_{n}, x, t\right) \leq 1$ for all $n \in \mathbb{N}$ and $t>0$ and hence Corollary 3.5(iii) holds. Thus all conditions of Corollary 3.5 are satisfied. The conclusion is that $T$ has a fixed point.

Remark 4.2 We observe that $T$ in Example 4.1 is not fuzzy $\psi$-contractive. Hence there exists a mapping which is fuzzy $\alpha-\psi$-contractive but not fuzzy $\psi$-contractive, although every fuzzy $\psi$-contractive mapping is obviously fuzzy $\alpha-\psi$-contractive.

In fact, set $\lambda=0.1$ and take $x=0.1, y=0.2$ in Example 4.1; we have $T x=x+\lambda=0.2$, $T y=y+\lambda=0.3$. Note that

$$
\begin{aligned}
& M(T x, T y, t)=M(0.2,0.3, t)=\frac{0.2}{0.3}=\frac{2}{3}, \\
& M(x, y, t)=M(0.1,0.2, t)=\frac{0.1}{0.2}=\frac{1}{2},
\end{aligned}
$$

and we have $M(T x, T y, t)<\psi(M(x, y, t))=\sqrt{\frac{1}{2}}$. This shows that $T$ is not fuzzy $\psi$-contractive.

Example 4.3 Let $X=[0, \infty)$ be endowed with the fuzzy metric $M(x, y, t)=\exp (-|x-y| / t)$ for all $x, y \in X$ and $t>0$. Let the single-valued mappings $T: X \rightarrow X$ be defined by

$$
T x= \begin{cases}\frac{1}{4} x^{2}, & x \in[0,1] \\ 2 x^{3}+1, & x \in(1, \infty)\end{cases}
$$

Define $\alpha, \eta: X \times X \times[0, \infty) \rightarrow X$ and $\psi \in \Psi$ by $\alpha \equiv 1$,

$$
\eta(x, y, t)=\left\{\begin{array}{ll}
1, & x \text { or } y \in[0,1], \\
\frac{1}{4}, & x, y \in(1, \infty),
\end{array} \quad t>0\right.
$$


and $\psi(s)=\sqrt{s}$ for $s \in[0,1]$, respectively. We prove that Corollary 3.8 can be applied to $T$. But the fuzzy $\psi$-contraction cannot be applied to $T$.

Proof Clearly, $(X, M, *)$ is a complete GV-space. We show that $T$ is an $\alpha-\eta$-admissible mapping. Let $x, y \in X$ and $t>0$; if $\eta(x, y, t) \geq 1$, then $x, y \in[0,1]$. On the other hand, for all $x \in[0,1]$, we have $T x \leq 1$. It follows that $\eta(T x, T y, t) \geq 1$. Also, $\eta(0, T 0, t) \geq 1$.

Now, if $\left\{x_{n}\right\}$ is a sequence in $X$ such that $\eta\left(x_{n}, x_{n+1}, t\right) \geq 1$, for all $n \in \mathbb{N}$ and $x_{n} \rightarrow x$ as $n \rightarrow \infty$, then $\left\{x_{n}\right\} \subset[0,1]$ and hence $x \in[0,1]$. This implies that $\eta\left(x_{n}, x, t\right) \geq 1$ for all $n \in \mathbb{N}$ and $t>0$. Let $\eta(x, y, t) \geq 1$. Then $x, y \in[0,1]$. We get

$$
M(T x, T y, t)=\exp \left(-\frac{\left|x^{2}-y^{2}\right|}{4 t}\right) \geq \exp \left(-\frac{|x-y|}{2 t}\right)=\psi(M(x, y, t)) .
$$

That is,

$$
\eta(x, y, t) \geq 1 \quad \Rightarrow \quad M(T x, T y, t) \geq \psi(M(x, y, t)) .
$$

Then all conditions of Corollary 3.8 hold. Hence, $T$ has a fixed point.

Let $x=2, y=3$ and $t>0$. Then $T x=17, T y=55$ and

$$
M(T x, T y, t)=\exp \left(-\frac{38}{t}\right)<\exp \left(-\frac{1}{t}\right)<\psi(M(x, y, t)) .
$$

This shows that the $\psi$-contraction introduced in [2] cannot be applied to $T$.

\section{Competing interests}

The author declares that they have no competing interests.

\section{Acknowledgements}

This work was supported by Natural Science Foundation of Zhejiang Province (LY12A01002).

Received: 6 October 2013 Accepted: 17 December 2013 Published: 10 Jan 2014

\section{References}

1. Gregori, V, Sapena, A: On fixed-point theorems in fuzzy metric spaces. Fuzzy Sets Syst. 125, 245-252 (2002)

2. Mihet, D: Fuzzy $\psi$-contractive mappings in non-Archimedean fuzzy metric spaces. Fuzzy Sets Syst. 159, 739-744 (2008)

3. Ćirić, LB: Some new results for Banach contractions and Edelstein contractive mappings on fuzzy metric spaces. Chaos Solitons Fractals 42, 146-154 (2009)

4. Radu, V: Some remarks on the probabilistic contractions on fuzzy Menger spaces. In: The Eighth International Conference on Appl. Math. Comput. Sci., Cluj-Napoca, 2002. Autom. Comput. Appl. Math. 11, 125-131 (2002)

5. Wang, S: Answers to some open questions on fuzzy $\psi$-contractions in fuzzy metric spaces. Fuzzy Sets Syst. 222, 115-119 (2013)

6. Wardowski, D: Fuzzy contractive mappings and fixed points in fuzzy metric spaces. Fuzzy Sets Syst. 222, 108-114 (2013)

7. Vetro, C: Fixed points in weak non-Archimedean fuzzy metric spaces. Fuzzy Sets Syst. 162, 84-90 (2011)

8. Zhu, X-H, Xiao, J-Z: Note on 'Coupled fixed point theorems for contractions in fuzzy metric spaces'. Nonlinear Anal. 74, 5475-5479 (2011)

9. Xiao, J, Zhu, X, Jin, X: Fixed point theorems for nonlinear contractions in Kaleva-Seikkala's type fuzzy metric spaces. Fuzzy Sets Syst. 200, 65-83 (2012)

10. Marudai, M, Srinivasan, PS: Some remarks on Heilpern's generalization of Nadler's fixed point theorem. J. Fuzzy Math. 12, 137-146 (2004)

11. Rezapour, S, Samei, M: Fixed points of some new contractions on intuitionistic fuzzy metric spaces. Fixed Point Theory Appl. 2013, 168 (2013)

12. Razani, A: A contraction theorem in fuzzy metric space. Fixed Point Theory Appl. 25, 257-265 (2005)

13. Qiu, Z, Hong, SH: Coupled fixed points for multivalued mappings in fuzzy metric spaces. Fixed Point Theory Appl. 2013, 162 (2013) 
14. Hong, SH, Peng, Y: Fixed points of fuzzy contractive set-valued mappings and fuzzy metric completeness. Fixed Point Theory Appl. 2013, 276 (2013)

15. Salimi, P, Latif, A, Hussain, N: Modified $\alpha$ - $\psi$-contractive mappings with applications. Fixed Point Theory Appl. 2013 $151(2013)$

16. Samet, B, Vetro, C, Vetro, P: Fixed point theorem for $\alpha$ - $\psi$-contractive type mappings. Nonlinear Anal. 75, 2154-2165 (2012)

17. Hussain, N, Salimi, P, Latif, A: Fixed point results for single and set-valued $\alpha-\eta$ - $\psi$-contractive mappings. Fixed Point Theory Appl. 2013, 212 (2013)

18. Hadžić, O, Pap, E: Fixed Point Theory in Probabilistic Metric Space. Kluwer Academic, Dordrecht (2001)

19. George, A, Veeramani, P: On some results in fuzzy metric spaces. Fuzzy Sets Syst. 64, 395-399 (1994)

20. Kramosil, I, Michálek, J: Fuzzy metrics and statistical metric spaces. Kybernetika 11, 326-334 (1975)

21. Gregori, V, Romaguera, S: Some properties of fuzzy metric spaces. Fuzzy Sets Syst. 115, 485-498 (2000)

22. Grabiec, M: Fixed points in fuzzy metric spaces. Fuzzy Sets Syst. 27, 385-389 (1989)

23. Vasuki, R, Veeramani, P: Fixed point theorems and Cauchy sequences in fuzzy metric spaces. Fuzzy Sets Syst. 135 , 415-417 (2003)

24. Rodríguez-Lôpez, J, Romaguera, S: The Hausdorff fuzzy metric on compact sets. Fuzzy Sets Syst. 147, $273-283$ (2004)

25. Gregori, V, Minana, J, Morillas, S: Some questions in fuzzy metric spaces. Fuzzy Sets Syst. 204, 71-85 (2012)

10.1186/1687-1812-2014-12

Cite this article as: Hong: Fixed points for modified fuzzy $\psi$-contractive set-valued mappings in fuzzy metric spaces Fixed Point Theory and Applications 2014, 2014:12

\section{Submit your manuscript to a SpringerOpen ${ }^{\circ}$ journal and benefit from:}

- Convenient online submission

- Rigorous peer review

- Immediate publication on acceptance

- Open access: articles freely available online

- High visibility within the field

- Retaining the copyright to your article 